\title{
Lost photon enhances superresolution
}

\author{
A. B. Mikhalychev $\mathbb{D}^{1 凶}$, P. I. Novik${ }^{1}$, I. L. Karuseichyk ${ }^{1}$, D. A. Lyakhov ${ }^{2}$ D. L. Michels ${ }^{2}$ and D. S. Mogilevtsev ${ }^{1}$
}

Quantum imaging can beat classical resolution limits, imposed by the diffraction of light. In particular, it is known that one can reduce the image blurring and increase the achievable resolution by illuminating an object by entangled light and measuring coincidences of photons. If an $n$-photon entangled state is used and the $n$ th-order correlation function is measured, the pointspread function (PSF) effectively becomes $\sqrt{n}$ times narrower relatively to classical coherent imaging. Quite surprisingly, measuring $n$-photon correlations is not the best choice if an $n$-photon entangled state is available. We show that for measuring ( $n-1)$-photon coincidences (thus, ignoring one of the available photons), PSF can be made even narrower. This observation paves a way for a strong conditional resolution enhancement by registering one of the photons outside the imaging area. We analyze the conditions necessary for the resolution increase and propose a practical scheme, suitable for observation and exploitation of the effect.

npj Quantum Information (2021)7:125; https://doi.org/10.1038/s41534-021-00465-4

\section{INTRODUCTION}

Diffraction of light limits the spatial resolution of classical optical microscopes $^{1,2}$ and hinders their applicability to life sciences at very small scales. Quite recently, a number of super resolving techniques, suitable for overcoming the classical limit, have been proposed. The approaches include, for example, stimulatedemission depletion microscopy ${ }^{3}$, super resolving imaging based on fluctuations ${ }^{4}$ or antibunched light emission of fluorescence markers ${ }^{5}$, structured illumination microscopy ${ }^{6,7}$, and quantum imaging $^{8-12}$.

Quantum entanglement is known to be a powerful tool for resolution and visibility enhancement in quantum imaging and metrology ${ }^{8-16}$. It has been shown that using $n$ entangled photons and measuring the $n$-th order correlations, one can effectively reduce the width of the point-spread function (PSF) $\sqrt{n}$ times $^{9,14,15,17}$ and beat the classical diffraction limit. The increase of the effect with the growth of $n$ can naively be explained as summing up the "pieces of information" carried by each photon when measuring their correlations. Such logic suggests that being given an $n$-photon entangled state, the intuitively most winning measurement strategy is to maximally exploit quantumness of the illuminating field and to measure the maximal available order of the photon correlations (i.e., the $n$th one).

Surprisingly, it is not always the case. First, it is worth mentioning that effective narrowing of the PSF and resolution enhancement can be achieved with classically correlated photons ${ }^{9}$ or even in the complete absence of correlations between fields emitted by different parts of the imaged object (as it is for the stochastic optical microscopy ${ }^{4}$ ). Moreover, the maximal order of correlations is not necessarily the best one ${ }^{18,19}$. In this contribution, we show that, for an entangled $n$-photon illuminating state, it is possible to surpass the measurement of all $n$-photon correlations by loosing a photon and measuring only $n-1$ remaining photons. According to our results, measurement of $(n-1)$ th-order correlations effectively leads to $\sqrt{2(n-1) / n}$ times narrower PSF and better resolution, quantified by Fisher information-based approach, than for commonly considered $n$-photon detection. It is even more strange in view of the notorious entanglement fragility $^{20}$ : if even just one of the entangled photons is lost, the correlations tend to become classical.

The insight for understanding that seeming paradox can be gained from a well-established ghost-imaging technique ${ }^{21-28}$ and from a more complicated approach of quantum imaging with undetected photons ${ }^{29-32}$. In both approaches, one of the entangled photons gains information about the object and "transmits" it through entanglement to the other photon, detected by a position-sensitive detector, by modifying its state. In our case, detecting $n-1$ photons and ignoring the remaining $n$th one effectively comprises two possibilities (see the imaging scheme depicted in Fig. 1): the $n$th photon can either fly relatively close to the optical axis of the imaging system towards the detector or go far from the optical axis and fail to pass through the aperture of the imaging system. In the first case, the photon can be successfully detected and provide us its piece of information. In the second case, it does not bring us the information itself, but effectively modifies the state of the remaining $n-1$ photons (as in refs. ${ }^{33-40}$ ). It effectively produces position-dependent phase shift, thus performing wave-function shaping ${ }^{37}$ and leading to an effect similar to structured illumination ${ }^{6,7}$, PSF shaping ${ }^{41}$, or linear interferometry measurement ${ }^{42}$, and enhancing the resolution. We show that for $n>2$ photons, the sensitivity-enhancement effect leads to higher information gain than just detection of the $n$th photon, and measurement of $(n-1)$-photon correlations surpasses $n$-photon detection. We refer to such $(n-1)$-photon detection as a "lost photon" case since one of the photons is ignored during the measurement.

The discussed sensitivity-enhancement effect can be used to increase resolution in practical imaging schemes. One can devise a conditional measurement set-up by placing a bucket detector outside the normal pathway of the optical beam (e.g., near the lens outside of its aperture) and post-selecting the outcomes when one photon gets to the bucket detector and the remaining $n-1$ ones successfully reach the position-sensitive detector, used for the coincidence measurements. We show that such a postselection scheme indeed leads to an additional increase of resolution relatively to $(n-1)$-photon detection. This is again an $n$-photon detection technique, but now inspired by the "lost photon" considerations and being more efficient than the

${ }^{1}$ B.I.Stepanov Institute of Physics, NAS of Belarus, Minsk, Belarus. ${ }^{2}$ Computer, Electrical and Mathematical Science and Engineering Division, 4700 King Abdullah University of Science and Technology, Thuwal, Saudi Arabia. ${ }^{凶}$ email: mikhalychev@gmail.com 


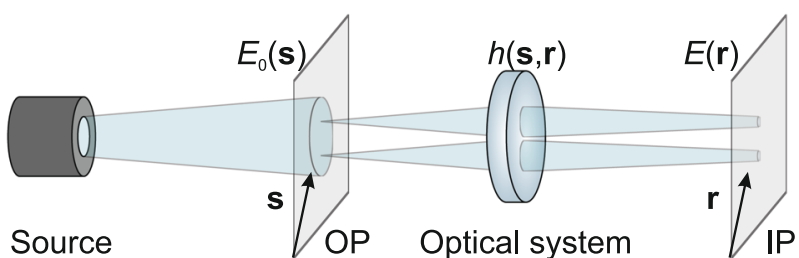

Fig. 1 General scheme of an imaging setup. See the text for details. A magnified or demagnified image of an object placed at the object plane OP is formed by the optical system at the image plane IP.

traditional measurement of the $n$th order correlations. Resolution enhancement by post-selecting the more informative field configuration is closely related to the spatial mode demultiplexing technique ${ }^{43-45}$. However, in our case, the selection of a more informative field part is performed by detection of a photon while all the remaining photons are detected in the usual way rather than by filtering the beam itself. Also, our technique bears some resemblance to the multi-photon ghost-imaging ${ }^{23}$.

Analysis of the PSF represents a fruitful approach for drawing useful conclusions based on our intuition, but, generally, it does not represent an accurate measure of resolution (see e.g., refs. $\left.{ }^{18,19}\right)$. For drawing quantitative conclusions about the resolution enhancement of the proposed technique relatively to traditional measurements of $n$ and $(n-1)$-photon coincidences, we employ the Fisher information, which has already proved itself as a powerful tool for analysis of quantum imaging problems and for meaningful quantification of resolution $18,19,41,43,44,46-49$. Our simulations show that for imaging a set of semi-transparent slits (i.e., for multi-parametric estimation problem), one indeed has a considerable increase in the information per measurement and the corresponding resolution enhancement. While the genuine demonstration of the discussed effects requires at least 3 entangled photons, which can be generated by a setup with complex nonlinear processes (e.g., cascaded spontaneous parametric down-conversion (SPDC) ${ }^{50}$, a combination of SPDC with upconversion $^{51}$, cascaded four-wave mixing ${ }^{52}$, or the third-order $\mathrm{SPDC}^{53-55}$ ), a relatively simple biphoton case is still suitable for observing resolution enhancement for a specific choice of the region where the $n$th photon (here, the second one) is detected.

\section{RESULTS}

\section{Imaging with entangled photons}

We consider the following common model of a quantum imaging setup (Fig. 1). An object is described by a transmission amplitude $A(\mathbf{s})$, where $\mathbf{s}$ is the vector of transverse position in the object plane. For simplicity's sake, we consider a commonly encountered case of an object with a real-valued transmission amplitude $0 \leq A$ (s) $\leq 1$ (see e.g., refs. ${ }^{9-12,23,25,28}$ ). It is illuminated by linearly polarized monochromatic light in an $n$-photon entangled quantum state

$$
\begin{aligned}
\left|\Psi_{n}\right\rangle & \propto \int d^{2} \mathbf{k}_{1} \cdots d^{2} \mathbf{k}_{n} \delta^{(2)}\left(\mathbf{k}_{1}+\cdots+\mathbf{k}_{n}\right) \\
& \times a^{+}\left(\mathbf{k}_{1}\right) \cdots a^{+}\left(\mathbf{k}_{n}\right)|0\rangle \propto \int d^{2} \mathbf{s}\left(a^{+}(\mathbf{s})\right)^{n}|0\rangle,
\end{aligned}
$$

where $a^{+}(\mathbf{k})$ and $a^{+}(\mathbf{s})$ are the operators of photon creation in the mode with the transverse wavevector $\mathbf{k}$ and at transverse position s, respectively. An optical system with the PSF (Green's function) $h$ $(\mathbf{s}, \mathbf{r})$ maps the object onto the image plane, where the field correlations are detected.

Features of the field passing through the analyzed object (and, thus, the object parameters) can be inferred from the measurement of intensity correlation functions accomplished by simple coincidence photo-counting. The detection rate of the $n$-photon coincidence at a point $\mathbf{r}$ is determined by the value of the $n$ th-order correlation function (see "Methods" section for details):

$$
G^{(n)}(\mathbf{r}) \propto\left|\int d^{2} \mathbf{s} A^{n}(\mathbf{s}) h^{n}(\mathbf{s}, \mathbf{r})\right|^{2} .
$$

The signal, described by Eq. (2), includes the $n$th power of the PSF, which is $\sqrt{n}$ times narrower than the PSF itself. At least for the object of just two transparent point-like pinholes, such narrowing yields $\sqrt{n}$ times the better visual resolution of the object than for imaging with coherent light (see e.g., refs. ${ }^{8,15}$ ).

Alternatively, one may try to ignore one of the photons and measure correlations of the remaining $(n-1)$ ones. The rate of $(n$ $-1)$-photon coincidences is described by the $(n-1)$ th-order correlation function:

$$
G^{(n-1)}(\mathbf{r}) \propto \int d^{2} \mathbf{s} A^{2(n-1)}(\mathbf{s})|h(\mathbf{s}, \mathbf{r})|^{2(n-1)} .
$$

Here, the $2(n-1)$ th power of the PSF is present. For $n>2$, the resolution enhancement factor $\sqrt{2(n-1)}$ is larger than the factor $\sqrt{n}$ achievable for $n$-photon detection. For $n=2$, we get $n=2(n-$ 1) and recover the common result (see also Fig. $2 f$ below): imaging with biphotons yields practically the same effective width of PSF as one would have for incoherent (thermal light) imaging (however, biphotons are advantageous if one is interested in the phase information about the object $)^{8,9,56,57}$. Notice that the resolution for imaging with biphotons $(n=2)$ is better than for the standard coherent imaging with uncorrelated photons $(n=1)^{8-11}$.

The result obtained looks quite counter-intuitive: each photon carries some information about the illuminated object while discarding one of the photons leads to additional information gain. This seeming contradiction is just a consequence of applying classical intuition to the quantum dynamics of an entangled system. Due to quantum correlations, an entangled photon can affect the state of the remaining ones and increase their "informativity" even when it is lost without being detected. Here we show that in our imaging scheme such an enhancement by loss is indeed taking place. Moreover, an additional resolution increase can be achieved through conditioning by detecting the photon outside the aperture of the imaging system (see Fig. 1).

\section{Effective state modification}

Let us consider the change of the $(n-1)$ photons state depending on the "fate" of the $n$th photon in more detail. We follow the approach discussed in ref. ${ }^{35}$, which consists of splitting the description of an $n$-photon detection process into 1-photon detection, density operator modification, and subsequent $(n-1)$ photon detection for the modified density operator. If we detect $(n-1)$-photon coincidence, the $n$th photon can be: (1) transmitted through the object into the imaging system aperture, (2) transmitted through the object outside the imaging system aperture, or (3) absorbed by the imaged object. Here, the "numbering" of photons has solely operational meaning: we are not aiming at distinguishing them, and the "nth photon" is not a particular photon, rather than just the last one remaining after $n-1$ photons have already been considered.

For the first possibility, the $n$th photon can reach the detector and potentially be registered at a certain point $\mathbf{r}^{\prime}$. The effective state of the remaining photons is (see "Methods" section):

$$
\left|\Psi_{n-1}^{(1)}\left(\mathbf{r}^{\prime}\right)\right\rangle \propto \int d^{2} \mathbf{s} A(\mathbf{s}) h\left(\mathbf{s}, \mathbf{r}^{\prime}\right)\left(a^{+}(\mathbf{s})\right)^{n-1}|0\rangle
$$

If we are interested in the detection of all the $n$ photons (i.e., post select the cases when the $n$th photon is successfully detected at the position $\mathbf{r}^{\prime}=\mathbf{r}$ ), the information gain due to the $n$th photon detection results from the factor $h(\mathbf{s}, \mathbf{r})$ introduced into the effective state (Eq. 4) of the remaining photons. It forces the photons to pass through the particular part of the object, which is 
a
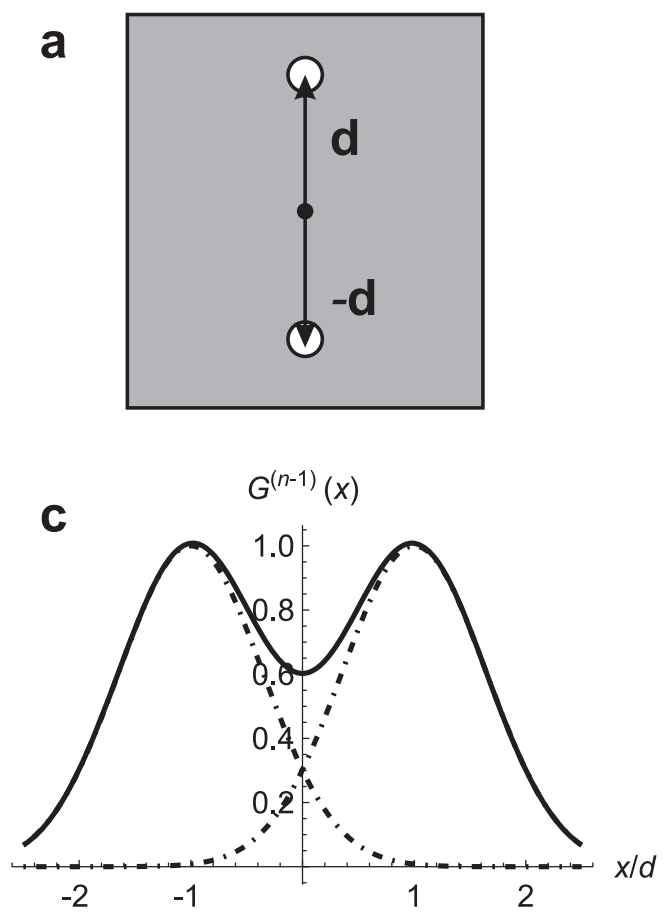

total signal

e

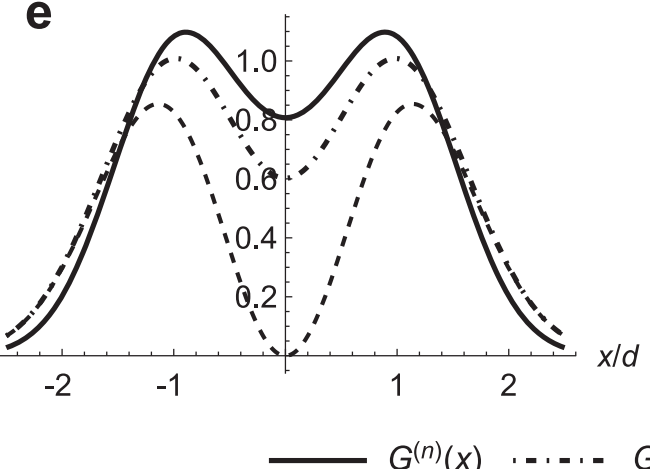

$\operatorname{signal}(x)$

$(x)=G^{(n-1)}(x)$

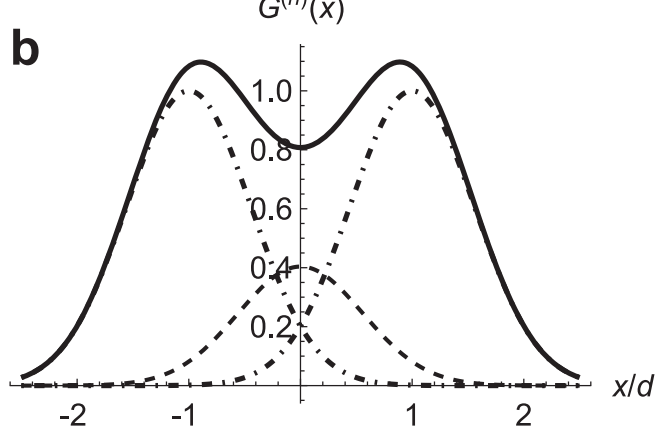

$G^{(n-1,1)}(x, k)$

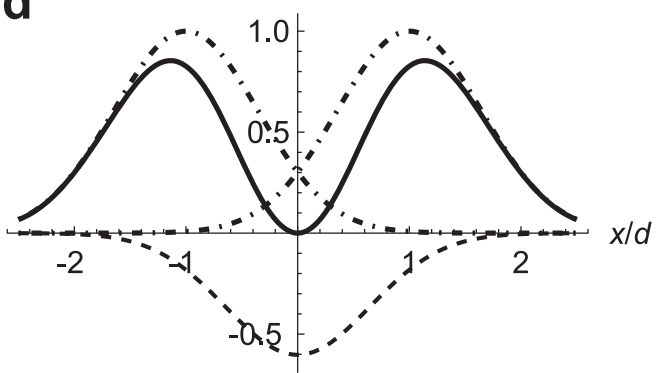

separate contributions ----- interference signal

Fig. 2 Model example. Imaging two pinholes (a), separated by the distance $2 d$, with the $n$-photon entangled state of light. The $n$-photon coincidence detection leads to constructive interference of the light passing through the pinholes (b). The interference is absent for ( $n-1)$ photon detection (c). For a particular value of the transverse momentum of one of the entangled photons, the conditional detection of the remaining $n-1$ photons may exhibit destructive interference (d). Dot-dashed lines represent separate contributions from the pinholes; the dashed line shows the interference signal; the solid line represents the sum of all the contributions. For the simulations, we used the PSF $h(\mathbf{s}, \mathbf{r}) \propto \exp \left[-(\mathbf{s}-\mathbf{r})^{2} /\left(5 d^{2}\right)\right]$ and $n=4$. Note, that the peaks in panels $\mathbf{c}$ and $\mathbf{d}$ are $n /(n-1)=4 / 3$ times wider than in panels $\mathbf{b}$, but the image contrast is better due to the absence of constructive interference. Panel e summarizes the results, showing the total signals from panels $\mathbf{b}$, $\mathbf{c}$, and $\mathbf{d}$ for their comparison: $G^{(n)}(x)$ (solid line), $G^{(n-1)}(x)$ (dot-dashed line), and $G^{(n-1,1)}(x)$ (dashed line). Panel $\mathbf{f}$ shows the same signals for $n=2$ and PSF $h(\mathbf{s}, \mathbf{r}) \propto \exp \left[-(\mathbf{s}-\mathbf{r})^{2} /\left(2.5 d^{2}\right)\right]$ with its width ensuring the same image contrast for the $n$th order correlations (shown by the solid line) as in previous panels. As expected for $n=2, G^{(n-1)}(x)$ does not outperform $G^{(n)}(x)$ in terms of the image contrast.

mapped onto the vicinity of the detection point $\mathbf{r}$, and effectively reduces the image blurring.

If, according to the second possibility, the $n$th photon goes outside the aperture of the imaging system and has the transverse momentum component $\mathbf{k}$, the affective state of the remaining photons is

$\left|\Psi_{n-1}^{(2)}(\mathbf{k})\right\rangle \propto \int d^{2} \mathbf{s} A(\mathbf{s}) \mathrm{e}^{\mathrm{ik} \cdot \mathbf{s}}\left(a^{+}(\mathbf{s})\right)^{n-1}|0\rangle$.
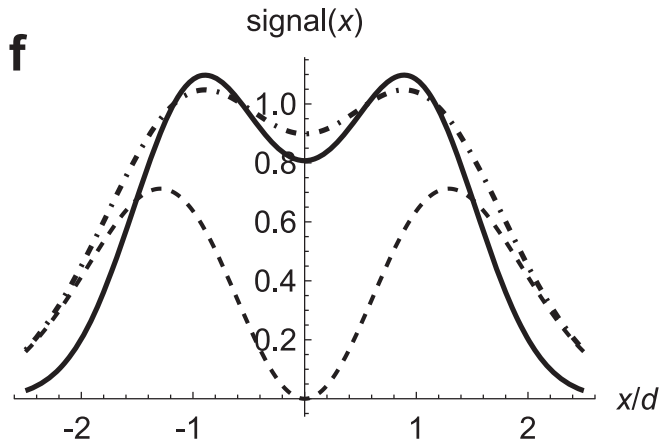

$G^{(n-1,1)}(x, k)$ 
density operator:

$\rho_{n-1}^{(3)}=\int d^{2} \mathbf{s}\left[1-A^{2}(\mathbf{s})\right]\left(a^{+}(\mathbf{s})\right)^{n-1}|0\rangle\langle 0|(a(\mathbf{s}))^{n-1}$.

By averaging over the three discussed possibilities (see "Methods" section), one can obtain the following expression for the effective state of the remaining $(n-1)$ photons:

$\rho_{n-1}=\int d^{2} \mathbf{s}\left(a^{+}(\mathbf{s})\right)^{n-1}|0\rangle\langle 0|(a(\mathbf{s}))^{n-1}$,

which is a separable (non-entangled, classically correlated) one: a mixture of states with $(n-1)$-photon excitations of spatial modes.

The detailed derivation of the result, while being quite trivial from a formal point of view, helps us to get to the following physical conclusions:

- The effective state of $n-1$ photons (and the $(n-1)$ th order correlation function) is modified, even if the $n$th photon is not detected by the observer, and depends on its "fate" (the way the photon actually passes). The "which path" information is generated due to the photon's interaction with its surrounding (the object, the detector, etc.), but maybe unavailable to the observer unless the two conditions are satisfied: (i) the photon successfully gets to the detector and (ii) the observer is measuring $n$-photon coincidences instead of ignoring the $n$th photon.

- The effective state of $n-1$ photons might be changed in a way, which provides the object resolution enhancement.

- When $n$-photon coincidences are measured, the $n$th photon detection actually leads to the postselection due to the discarding of possibilities leading to the photon loss.

- For $n>2$, the advantage gained from registering more photon coincidences with the $n$th photon detection does not compensate for the information loss caused by discarding outcomes corresponding to the strongly modified $(n-1)$ photon state, which is more sensitive to the object features (see Eqs. (2) and (3)).

Further, we discuss how the advantageous outcomes can be postselected, instead of being discarded, for resolution enhancement.

\section{Model example}

To gain a better understanding of the processes of resolution enhancement by a photon loss and postselection, let us consider a standard model object illuminated by an $n$-photon entangled state and consisting of two pinholes, which are separated by the distance $2 d$ and positioned at the points $\mathbf{d}$ and $-\mathbf{d}$ (Fig. 2a). If the pinholes are small enough, the light passing through the object can be decomposed into just two field modes, corresponding to the spherical waves emerging from the two pinholes and further denoted by the indices "+" and "-" for the upper and the lower pinhole, respectively. For simplicity's sake, we assume that the PSF is a real-valued function and that the light state directly after the object has the form of a NOON-state of the discussed modes "+" and "-":

$$
\left|\Phi_{n}\right\rangle \propto|n\rangle_{+}|0\rangle_{-}+|0\rangle_{+}|n\rangle_{-} \text {. }
$$

The $n$ th-order correlation signal contains separate contributions from the single pinholes and a cross-term, caused by constructive interference and leading to an additional blurring of the image (Fig. 2b):

$$
G^{(n)}(\mathbf{r}) \propto h^{2 n}(\mathbf{d}, \mathbf{r})+h^{2 n}(-\mathbf{d}, \mathbf{r})+2 h^{n}(\mathbf{d}, \mathbf{r}) h^{n}(-\mathbf{d}, \mathbf{r}) .
$$

While the scheme in Fig. 1 with the object from Fig. 2a may resemble the classical double-slit interference experiment, it contains a lens, which effectively ensures near-field imaging by compensating any phase difference introduced by the spatial separation of the considered pinholes. In the resulting image, the interference is governed by the phases of the input light only and remains constructive for any separation $d$ if the light state is given by Eq. (8).

The $(n-1)$ th-order correlations include only separate singlepinhole signals and produce a sharper image for $n>2$ (Fig. 2c):

$$
G^{(n-1)}(\mathbf{r}) \propto h^{2(n-1)}(\mathbf{d}, \mathbf{r})+h^{2(n-1)}(-\mathbf{d}, \mathbf{r})
$$

Let us interpret these results in terms of detecting $n-1$ photons conditioned by the $n$th photon detection. According to Eq. (4), if the photon is detected at the point $\mathbf{r}$ of the image plane, it transforms the state of the remaining photons into

$$
\begin{aligned}
\left|\Phi_{n-1}^{(1)}(\mathbf{r})\right\rangle & \propto h(\mathbf{d}, \mathbf{r})|n-1\rangle_{+}|0\rangle_{-} \\
& +h(-\mathbf{d}, \mathbf{r})|0\rangle_{+}|n-1\rangle_{-} .
\end{aligned}
$$

The state coherence is preserved, while the blurring, caused by constructive interference, is slightly reduced due to certain "which path" information provided by the $n$th photon detection.

If the $n$th photon is characterized by the transverse momentum $\mathbf{k},|\mathbf{k}|>k_{\max }$, and does not get into the imaging system aperture, the effective modified state of the remaining photons is (see Eq. (5)):

$$
\begin{aligned}
& \left|\Phi_{n-1}^{(2)}(\mathbf{k})\right\rangle \propto \mathrm{e}^{\mathbf{i} \mathbf{k} \cdot \mathbf{d}}|n-1\rangle_{+}|0\rangle_{-} \\
& +\mathrm{e}^{-i \mathbf{k} \cdot \mathbf{d}}|0\rangle_{+}|n-1\rangle_{-},
\end{aligned}
$$

Now, the phase shift between the two modes depends on $\mathbf{k}$ and can lead to destructive interference, which enhances the contrast of the image. For example, when $\mathbf{k} \cdot \mathbf{d}=\pi / 2$, one has maximally destructive interference and the detected signal is proportional to $\left|h^{(n-1)}(\mathbf{d}, \mathbf{r})-h^{(n-1)}(-\mathbf{d}, \mathbf{r})\right|^{2}$ with $100 \%$ visibility of the gap between the two peaks (Fig. 2d). Such an advantageous situation can be postselected by placing an additional detector in the farfield outside the aperture in the direction $\mathbf{k} /|\mathbf{k}|$ from the object.

Discarding the information about the $n$th photon (measuring $\left.G^{(n-1)}\right)$ corresponds to averaging over the possibilities to have the photon passing to the detector and missing it, and yields the following mixed state of the remaining photons:

$$
\begin{aligned}
\rho_{n-1} & \propto|n-1\rangle_{+}\langle n-1|\otimes| 0\rangle_{-}\langle 0| \\
& +|0\rangle_{+}\langle 0|\otimes| n-1\rangle_{-}\langle n-1| .
\end{aligned}
$$

I.e., the cross-terms with constructive and destructive interference cancel each other, and the resulting mixed state allows for some resolution gain over the pure $n$th photon NOON state.

\section{Application to quantum imaging}

To illustrate the possible application of the ideas to practical quantum imaging, we consider an object represented by a set of semitransparent slits (Fig. 3a, c). The resolution of the modeled optical system is limited by diffraction at the lens aperture, which admits only the photons with the transverse momentum $\mathbf{k}$ not exceeding $k_{\max }:|\mathbf{k}| \leq k_{\max }$.

We compare the following three strategies: (i) measuring $G^{(n)}(\mathbf{r})$ along the direction perpendicular to the slits (the signal is described by Eq. (2)); (ii) measuring $G^{(n-1)}(\mathbf{r})$ at the same points (Eq. (3)); (iii) measuring coincidence signal $G^{(n-1,1)}(\mathbf{r}, \Omega)$ of $n-1$ photons detected at the point $\mathbf{r}$ of the detection plane and the $n$th photon being anywhere in certain region $\Omega$ outside the lens aperture. For the latter case, the signal can be written as

$$
\begin{aligned}
G^{(n-1,1)}(\mathbf{r}, \Omega) & \propto \int d^{2} \mathbf{s} d^{2} \mathbf{s}^{\prime} A^{n}(\mathbf{s}) A^{n}\left(\mathbf{s}^{\prime}\right) \\
& \left.\times h^{n-1}(\mathbf{s}, \mathbf{r}) h^{n-1}\left(\mathbf{s}^{\prime}, \mathbf{r}\right)\right) g\left(\mathbf{s}-\mathbf{s}^{\prime}\right),
\end{aligned}
$$

where

$g\left(\mathbf{s}-\mathbf{s}^{\prime}\right)=\int_{\mathbf{k} \in \Omega} d^{2} \mathbf{k} \mathrm{e}^{\mathrm{i} \mathbf{k} \cdot\left(\mathbf{s}-\mathbf{s}^{\prime}\right)}$. 
a

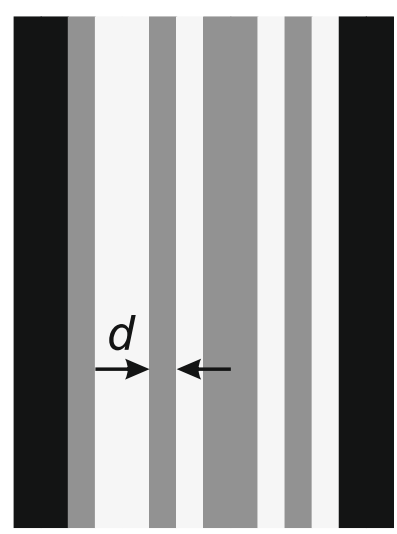

C

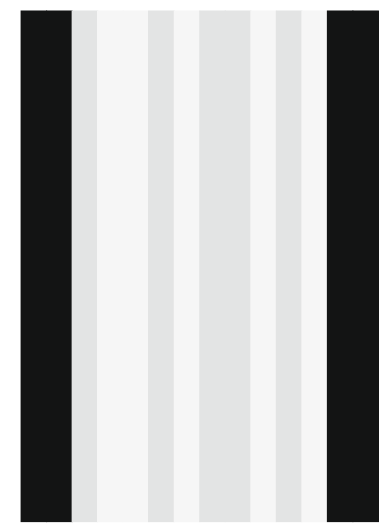

b

Signal $(x)$

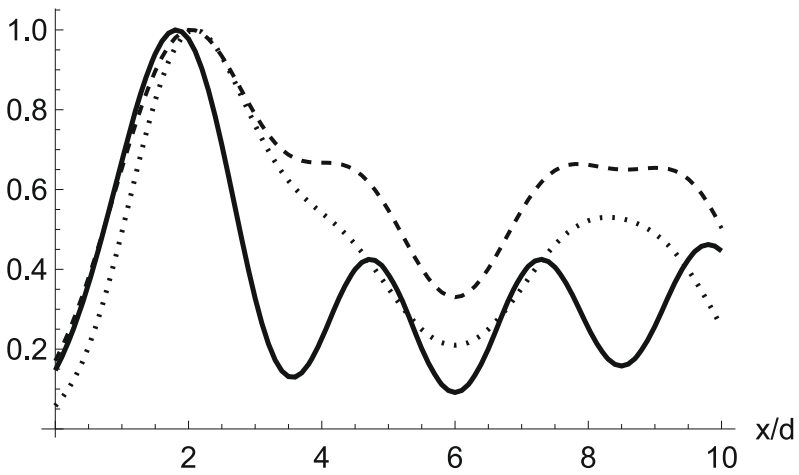

Signal $(x)$

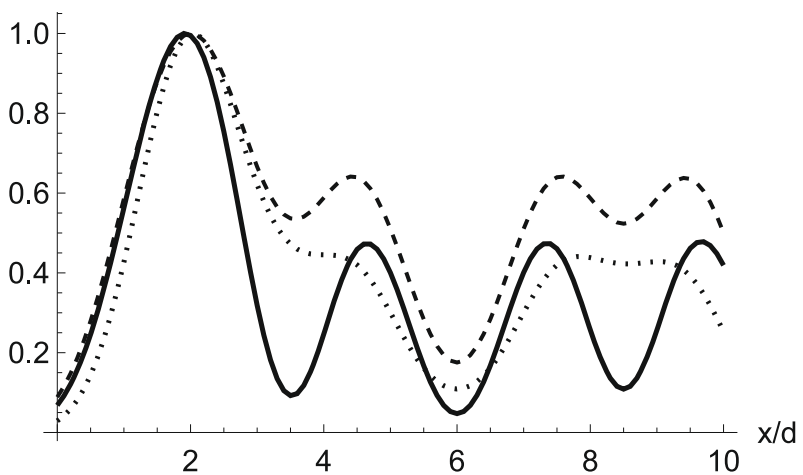

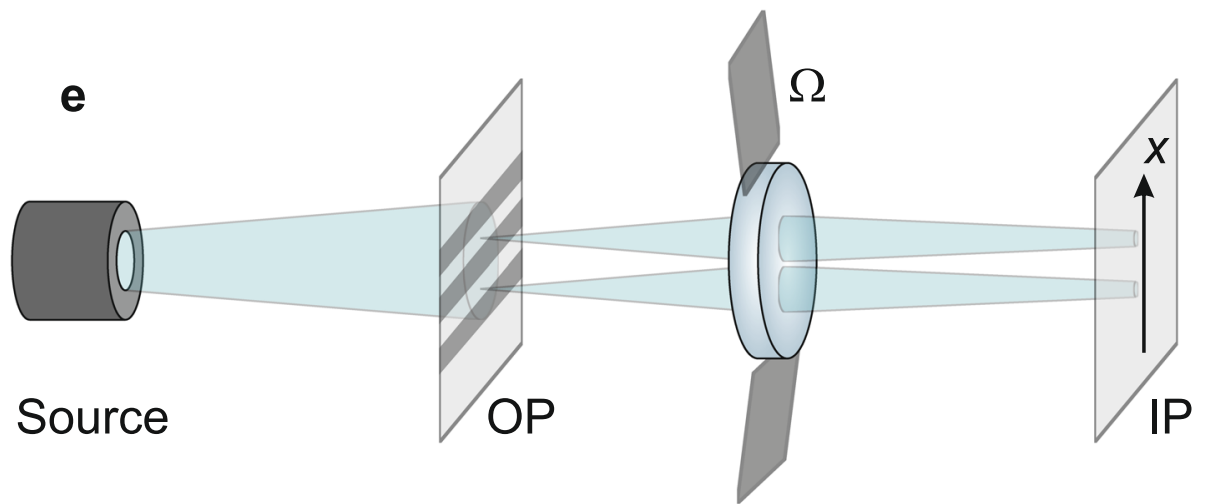

Fig. 3 Application of the resolution-enhancement ideas to a realistic setup. Model objects (a, c), imaging scheme for ( $n-1)$-photon coincidence conditioned by detecting a photon in the region $\Omega(\mathbf{e})$, and simulation results (b, d). Sets of semitransparent slits with transmission amplitudes $0.5 \div 1$ (a) and $0.9 \div 1$ (c) were used as model objects. The signal $\left(G^{(n)}\right.$ _dotted lines, $G^{(n-1)}$-dashed lines, $G^{(n-1,1)}-$ solid lines) was simulated for the object from panel $\mathbf{a}, k_{\max } d=1$, and $n=3$ (b) and 4 (d). The detection region for the $n$th photon is $\Omega=\left\{\mathbf{k}: k_{\max } \leq|\mathbf{k}| \leq 2 k_{\max }\right\}$. The axis $x$ is directed across the slits. The coordinate $x$ is normalized by the slit size $d$ indicated in plot a.

Integration in Eq. (15) corresponds to detection of the $n$th photon by a bucket detector, similarly to multiphoton ghost imaging ${ }^{23-25}$. The difference is that the remaining $n-1$ photons do also pass through the investigated object before getting to the position-resolving detector in our case. The scheme can also be considered as a generalization of hybrid near-field and far-field imaging when the entangled photons are analyzed partially in position space and partially in momentum space. Note, that $G^{(n-1,1)}$ does not turn into $G^{(n)}$ even in the limiting case when the region $\Omega$ shrinks to a single point: the first case corresponds to farfield detection of the $n$th photon (i.e., $\Omega$ defines a point in $\mathbf{k}$-space, not a particular position $\mathbf{r}$ ), while in the latter case all the $n$ photons are localized in the position space by the detector.

Simulated images are shown in Fig. 3b, d. One can clearly see that $(n-1)$-photon detection yields better visual resolution than measurement of the $n$ th-order coincidences, while $G^{(n-1,1)}$ provides additional contrast enhancement. While using a narrower region $\Omega$ may additionally increase the contrast of the image and the information content per a single detection event (see Fig. 4 below), it also reduces the number of detected coincidences (see Fig. 5). On the other hand, even a relatively large bucket detector, used for the shown simulations, is sufficient for noticeable enhancement of resolution.

Of course, the effective narrowing of the PSF by measuring correlation functions does not necessarily mean a corresponding increase in precision of inferring of the analyzed parameters (positions of the object details, channel characteristics, etc.) ${ }^{18,19}$. However, at least for certain imaging tasks (such as, for example, a cornerstone problem of finding a distance between two point sources in the far-field imaging), narrowing of the PSF can indeed 

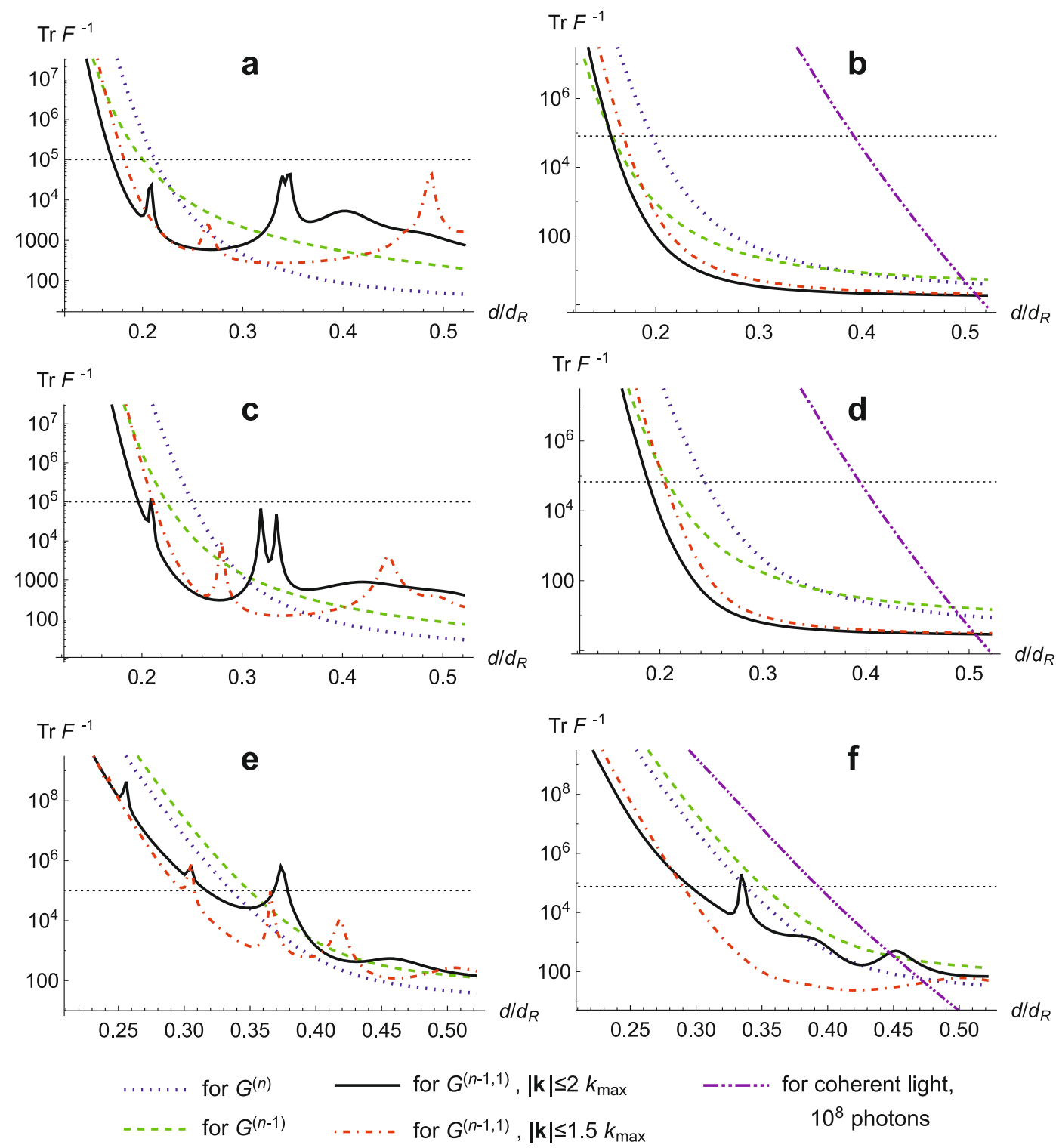

f

$$
\begin{aligned}
& \ldots \ldots \ldots \text { for } G^{(n)} \quad-\text { for } G^{(n-1,1)},|\mathbf{k}| \leq 2 k_{\max } \\
& \ldots \ldots \text { for } G^{(n-1)} \quad \ldots \ldots \text { for } G^{(n-1,1)},|\mathbf{k}| \leq 1.5 k_{\max }
\end{aligned}
$$

Fig. 4 Dependence of the trace of inverse FIM on the normalized slit size $\mathbf{d} / \mathbf{d R}$. The results are shown for detection of $n$ th-order correlations (blue dotted lines), $(n-1)$ th-order correlations (green dashed lines), and $(n-1)$ th-order correlations, conditioned by detection of the $n$th photon in the region $\Omega=\left\{\mathbf{k}: k_{\max } \leq|\mathbf{k}| \leq 2 k_{\max }\right\}$ (black solid lines) and $\Omega=\left\{\mathbf{k}: k_{\max } \leq|\mathbf{k}| \leq 1.5 k_{\max }\right\}$ (red dot-dashed lines) for $n=4$ (a, $\mathbf{b}$ ), $3(\mathbf{c}, \mathbf{d})$, and $2(\mathbf{e}, \mathbf{f})$. The objects, shown in Fig. 3a (for plots $\mathbf{a}, \mathbf{c}$, and $\mathbf{e}$ ) and Fig. 3c (for plots $\mathbf{b}$, d, and f), were decomposed in terms of 10 basis functions, representing slit-like pixels with equal widths $d$. The object size, the basis functions widths, and the step of the signal sampling points were scaled in the same way with $d$. Horizontal dotted lines indicate the threshold $\operatorname{Tr} F^{-1} \leq N_{\max }=10^{5}$, used for quantification of resolution. For comparison, panels $\mathbf{b}, \mathbf{d}, \mathbf{f}$ also show (by double-dot-dashed purple lines) the trace of inverse FIM for traditional coherent-light imaging for detection of $10^{8}$ photons (instead of a single coincidence event for all other lines).

lead to an increase of the informational content per measurement, and to the potentially unlimited resolution with increasing of $n^{19}$.

To describe the resolution enhancement in a quantitative and more consistent way, we employ Fisher information. Let the transmission amplitude of the object be decomposed as $A(\mathbf{s})=\Sigma_{\mu} \theta_{\mu} f_{\mu}(\mathbf{s})$, where the basis functions $f_{\mu}(\mathbf{s})$ can represent e.g., slit-like pixels for the considered example ${ }^{47}$. Then the problem of finding $A(\mathbf{s})$ becomes equivalent to the reconstruction of the unknown decomposition coefficients $\theta_{\mu}$. If one has a certain signal $S(\mathbf{r})$, sampled at the points $\left\{\mathbf{r}_{i}\right\}$, Fisher information matrix (FIM $^{58,59}$, normalized by a single detection event, can be introduced as

$F_{\mu \nu}=\sum_{i}\left(\frac{1}{S\left(\mathbf{r}_{i}\right)} \frac{\partial S\left(\mathbf{r}_{i}\right)}{\partial \theta_{\mu}} \frac{\partial S\left(\mathbf{r}_{i}\right)}{\partial \theta_{v}}\right) / \sum_{i} S\left(\mathbf{r}_{i}\right)$.
Here, the "detection event" corresponds to the registration of a coincidence signal of $n$ or $n-1$ photons (depending on the measurement type) within the specified time frame, rather than the detection of every single photon.

Cramér-Rao inequality ${ }^{59,60}$ bounds the total reconstruction error (the sum of variances of the estimators for all the unknowns $\left\{\theta_{\mu}\right\}$ ) by the trace of the inverse of FIM:

$\Delta^{2}=\sum_{\mu} \Delta \theta_{\mu} \geq \frac{1}{N} \operatorname{Tr}^{-1}$,

where $N$ is the number of registered coincidence events. When the size of the analyzed object features (e.g., the slit size $d$ in Fig. 3a) tends to zero, the bound in Eq. (17) diverges (the effect is termed "Rayleigh's curse"). The achievable resolution can, therefore, be determined by the feature size $d$, which $\operatorname{TrF}^{-1}$ starts 


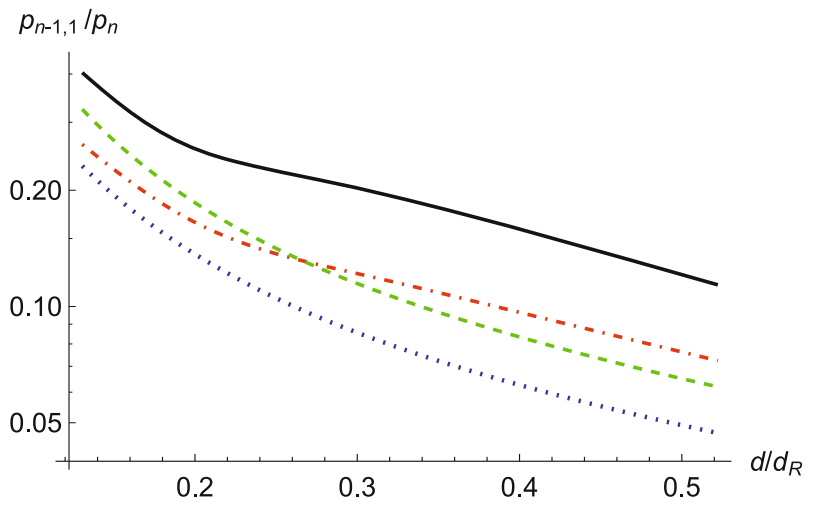

Fig. 5 Dependence of the overall detection probabilities ratio on the normalized slit size $\boldsymbol{d} / \boldsymbol{d}_{\mathrm{R}}$ (see details in the text). The $n$th photon is detected in the region $\Omega=\left\{\mathbf{k}: k_{\max } \leq|\mathbf{k}| \leq 2 k_{\max }\right\}$ (solid black and dashed green lines) or $\Omega=\left\{\mathbf{k}: k_{\max } \leq|\mathbf{k}| \leq 1.5 k_{\max }\right\}$ (dot-dashed red and dotted blue lines) for $n=4$. The objects are shown in Fig. 3a (for solid black and dot-dashed red lines) and Fig. $3 c$ (for dashed green and dotted blue lines).

growing rapidly with the decrease of $d$. A more rigorous definition can be given by specifying a certain reasonable threshold $N_{\max }$ for the maximal required number of registered coincidence events $N$ (e.g., we take $N_{\max }=10^{5}$ for further examples) and imposing the restriction $\mathrm{Tr}^{-1} \leq N_{\max }$ (see "Methods" section).

The dependence of the predicted reconstruction error on the normalized object scale $d / d_{\mathrm{R}}$ (where $d_{\mathrm{R}}=3.83 / k_{\max }$ is the classical Rayleigh limit for the considered optical system) is shown in Fig. 4. The sampling points for the signal are taken with the step $d / 2$ along a line perpendicular to slits. As expected, ignoring $n$th photon and measuring $(n-1)$-photon coincidences brings about larger achievable information per measurement, and correspondingly lower errors in object parameter estimation, yielding $(10 \div 20) \%$ better resolution for $n=3$ and 4 . According to the theoretical predictions, for $n=2$ no resolution increase is observed.

Also, our results confirm that the proposed hybrid scheme is indeed capable of increasing the resolution for $n=3$ and 4 by conditioned detection of the $n$th photon. Additional information that can be gained relative to the measurement of $(n-1)$ photon correlations is about $(10 \div 15) \%$. However, that gain vanishes in the regime of deep superresolution $\left(d \lesssim 0.2 d_{R}\right)$ : the black solid and red dot-dashed lines intersect with the green dashed one for small $d / d_{\mathrm{R}}$ in Fig. 4. The reason for such behavior is that the effective phase shift, introduced by detection of the $n$th photon with the transverse momentum $\mathbf{k}$ outside of the aperture of the imaging system, becomes insufficient for the resolution enhancement for $|\mathbf{k}| d \ll 1$.

For $n=2$, the scheme also can give certain advantages (Fig. 4e, f), which, however, are not so prominent because they do not stem from the fundamental requirement of having a better resolution for $G^{(n-1)}$ than for $G^{(n)}$. Still, taking into account the difficulties in the generation of 3-photon entangled states ${ }^{50-55}$, an experiment with biphotons can be proposed for initial tests of the approach.

The plots, shown in Fig. 4, represent information per single coincidence detection event. Therefore, certain concerns about the rates of such events may arise: waiting for a highly informative, but very rare event can be impractical. Fig. 5 shows the ratio of the overall detection probabilities $p_{n-1,1} / p_{n}$, where $p_{n-1,1}$ corresponds to $(n-1)$-photon coincidence, conditions by detection of a photon outside the aperture, and $p_{n}$ describes the traditional measurement of $n$-photon coincidences. For a signal $S$ $\left(\mathbf{r}_{i}\right)$, the overall detection probability is defined as $p=\Sigma_{i} S\left(\mathbf{r}_{i}\right)$ and represents the denominator of Eq. (16). When plotting Fig. 5, we do not include the measurement of $G^{(n-1)}$ in the comparison, because the ratio of probabilities for $(n-1)$ and $n$-photon detection events strongly depends on details of a particular experiment, such as the efficiency of the detectors.

The rate of $(n-1)$-photon coincidences, conditioned by the detection of a photon outside of the aperture, is indeed $3 \div 20$ times smaller than the rate of $n$-photon coincidences. However, for the considered multi-parametric problem, the "Rayleigh curse" leads to a very fast decrease of information when the slit size $d$ becomes smaller than the actual resolution limit, and the effect of rate difference is almost negligible. For example, for $n=4$, the object, shown in Fig. 3a, and the threshold $\mathrm{TrF}^{-1} \leq 10^{5}$, the minimal slit width $d$ for successful resolution of transmittances equals $0.212 d_{R}$ for the measurement of $G^{(n)}$, $0.170 d_{R}$ for the measurement of $G^{(n-1,1)}$ with the $n$th photon detected in the region $\Omega=\left\{\mathbf{k}: k_{\max } \leq|\mathbf{k}| \leq 2 k_{\max }\right\}$, and $0.177 d_{\mathrm{R}}$ for the same measurement of $G^{(\bar{n}-1,1)}$ when the reduced detection rate is taken into account. Notice, that all the mentioned values of the resolved feature size $d$ are quite far beyond the classical resolution limit $d_{R}$.

At the first glance, the reported percentage of the resolution enhancement does not look very impressive or encouraging. However, one should keep in mind that the increase of the number of entangled photons from $n=2$ to $n=3$, while requiring significant experimental efforts, leads to the effective PSF narrowing just by $22 \%$ for the measurement of $G^{(n)}$. The transition from a 3-photon entangled state to a 4-photon one yields only $15 \%$ narrower PSF. Moreover, the actual resolution enhancement is typically smaller than the relative change of the PSF width ${ }^{18,19}$, especially for high orders of the correlations, where it may saturate completely. The proposed approach provides a similar magnitude of the resolution increase on the cost of adding a bucket detector to the imaging scheme, which is much simpler than changing the number of entangled photons.

A similar concern about the soundness of the results may be elicited by recalling a simple problem of resolving two-point sources, commonly investigated theoretically $41,43,48$. For such a simple model situation, the error of inferring the distance $d$ between the sources scales as $\Delta d \propto d^{-1} N^{-1 / 241}$, where $N$ is the number of detected events. Therefore, to resolve twice smaller separation of the two sources with the same error, one just needs to perform a 4 times longer experiment and collect $4 \mathrm{~N}$ events. The situation becomes completely different when a more practical multiparametric problem is considered ${ }^{47}$ : the achievable resolution becomes practically insensitive to the data acquisition time (as soon as the number of detected events becomes sufficiently large). For example, for the situation described by the solid black line in Fig. 4a, a 100-fold increase of the acquisition time leads to a $14 \%$ larger resolution. Moreover, as one can see from the doubledot-dashed purple lines in Fig. $4 b, c$, d, traditional coherent-light imaging with intensity detection does not provide sufficient information about the object features in the superresolution regime $\left(d \lesssim 0.4 d_{R}\right)$ even for $10^{8}$-times increase of the number of detected photons. That observation indicates that appropriately used entangled photons sources can outperform classical light sources, which are brighter even by many orders of magnitude. The effect is especially important for biological samples, vulnerable to photodestruction.

\section{DISCUSSIONS}

We have demonstrated how to enhance the resolution of imaging with an $n$-photon entangled state by loosing a photon and measuring the $(n-1)$ th-order correlation function instead of the $n$ th-order coincidence signal. The resolution gain occurs despite the breaking of entanglement as a consequence of the photon loss. We have explained the effect in terms of the effective modification of the remaining photons state when one of the 
entangled photons is lost. Measurement of $(n-1)$-photon coincidences for an $n$-photon entangled state not only discards some information carried by the ignored $n$th photon but also makes the resulting signal more informative in the considered imaging experiment. The latter effect prevails for $n>2$ and leads to an increase of the information per measurement and to decrease of the lower bounds for the object inference errors.

The $(n-1)$-photon detection represents a mixture of different possible outcomes for the discarded $n$th photon, including its successful detection at the image plane (resulting in the $n$-photon coincidence signal). The information per a single $(n-1)$-photon detection event is averaged over the discussed possibilities and, for $n>2$, is larger than the information for a single $n$-photon coincidence event. It means that certain outcomes for the $n$th photon provide more information per event than the average value, achieved for $G^{(n-1)}$. We prove that proposition constructively by proposing a hybrid measurement scheme, which provides resolution increase relatively to detection of $(n-1)$ photon coincidences. Intentional detection of a photon outside the optical system, used for imaging of the object, introduces an additional phase shift and increases the sensitivity of the measurement performed with the remaining photons. Our simulations show that the effect can be observed even for $n=$ 2 , thus making its practical implementation much more realistic. We believe that our observation will pave a way for practical exploitation of entangled states by devising a super resolving imaging scheme conditioned on detecting photons not only successfully passing through the imaging system, but also those missing it.

\section{METHODS}

\section{Expressions for field correlation functions}

For the imaging setup, shown in Fig. 1, the positive-frequency field operators $E(\mathbf{r})$ at the detection plane are connected to the operators $E_{0}(\mathbf{s})$ of the field illuminating the object as

$E^{(+)}(\mathbf{r})=\int d^{2} \mathbf{s} E_{0}^{(+)}(\mathbf{s}) A(\mathbf{s}) h(\mathbf{s}, \mathbf{r})$.

The $n$ th-order correlation function for the $n$-photon entangled state (Eq. 1) is calculated according to the following standard definition:

$G^{(n)}(\mathbf{r})=\left\langle\Psi_{n}\left|\left[E^{(-)}(\mathbf{r})\right]^{n}\left[E^{(+)}(\mathbf{r})\right]^{n}\right| \Psi_{n}\right\rangle$

where $E^{(-)}(\mathbf{r})=\left[E^{(+)}(\mathbf{r})\right]^{+}$is the negative-frequency field operator. By substitution of Eq. (18) into Eq. (19), one can obtain the expression (Eq. 2) in the Results section.

The $(n-1)$ th-order correlation function is calculated according to the expression

$G^{(n-1)}(\mathbf{r})=\left\langle\Psi_{n}\left|\left[E^{(-)}(\mathbf{r})\right]^{n-1}\left[E^{(+)}(\mathbf{r})\right]^{n-1}\right| \Psi_{n}\right\rangle$,

which yields Eq. (3) after substitution of Eq. (18).

\section{Effective $(n-1)$-photon state}

The density operator, describing the effective $(n-1)$-photon state averaged over the possible "fates" of the $n$th photon, discussed in the main text, is

$\rho_{n-1}=\rho_{n-1}^{(1)}+\rho_{n-1}^{(2)}+\rho_{n-1}^{(3)}$,

where the operators $\rho_{n-1}^{(k)}$ are indexed according to the introduced possibilities and normalized in such a way that $\operatorname{Tr} \rho_{n-1}^{(k)}$ is the probability of the $k$ th "fate".

According to the approach, discussed in ref. ${ }^{35}$, detection of the $n$th photon at the position $\mathbf{r}^{\prime}$ of the detector effectively modifies the states of the remaining $(n-1)$ photons in the following way:

$\left|\Psi_{n-1}^{(1)}\left(\mathbf{r}^{\prime}\right)\right\rangle \propto \mathrm{e}^{(+)}\left(\mathbf{r}^{\prime}\right)\left|\Psi_{n}\right\rangle$

Substitution of Eqs. (1) and (18) yields Eq. (4).
If we ignore the information about the position $\mathbf{r}^{\prime}$ of the photon detection, the contribution to the averaged density operator (Eq. 21) is

$\rho_{n-1}^{(1)}=\int d^{2} \mathbf{r}^{\prime}\left|\Psi_{n-1}^{(1)}\left(\mathbf{r}^{\prime}\right)\right\rangle\left\langle\Psi_{n-1}^{(1)}\left(\mathbf{r}^{\prime}\right)\right|$.

For the possibility, described by Eq. (5) and corresponding to the $n$th photon passage outside the aperture of the imaging system, the contribution to the averaged density operator (Eq. 21) is

$\rho_{n-1}^{(2)}=\int_{|\mathbf{k}|>k_{\max }} d^{2} \mathbf{k}\left|\Psi_{n-1}^{(2)}(\mathbf{k})\right\rangle\left\langle\Psi_{n-1}^{(2)}(\mathbf{k})\right|$,

where $k_{\max }$ is the maximal transverse momentum transferred by the optical system: $k_{\max }=k R / s_{0} ; k$ is the wavenumber of the light, $R$ is the radius of the aperture, and $s_{0}$ is the distance between the object and the lens used for imaging.

Calculating integrals in Eqs. (23), (24), and (6), and taking into account the connection between the PSF shape and $k_{\max }$ (see e.g., ref. ${ }^{8}$ ), one can obtain Eq. (7) for the effective $(n-1)$-photon state.

\section{Model of the point-spread function}

For the simulations, illustrated by Figs. 3, 4, and 5, we assume for simplicity that the magnification of the optical system is equal to 1 , neglect the phase factor in PSF, and use the expression

$$
\begin{aligned}
h(\mathbf{s}, \mathbf{r}) & =\int_{|\mathbf{k}| \leq k_{\max }} d^{2} \mathbf{k} \mathrm{e}^{\mathbf{i k \cdot} \cdot(\mathbf{s}+\mathbf{r})} \\
& =2 \pi k_{\max }^{2} \operatorname{somb}\left(k_{\max }|\mathbf{s}+\mathbf{r}|\right),
\end{aligned}
$$

where $\operatorname{somb}(x)=2 J_{1}(x) / x, J_{1}(x)$ is the first-order Bessel function and $k_{\max }$ is the maximal transverse momentum transferred by the optical system.

\section{Quantification of resolution}

Let us assume that a reasonable number of detected coincidence events $N$ in a quantum imaging experiment is limited by the value $N_{\max }$ and the acceptable total reconstruction error (see Eq. (17)) is $\Delta^{2} \leq 1$. Therefore, Eq. (17) implies the following threshold for the trace of the inverse of FIM: $\operatorname{TrF}^{-1} \leq N \Delta^{2} \leq N_{\max }$.

Therefore, one can define the spatial resolution, achievable under the described experimental conditions, as the minimal feature size $d$, for which the condition (Eq. 26) is satisfied.

\section{DATA AVAILABILITY}

The datasets generated and analyzed during this study are available from the corresponding author on reasonable request.

Received: 4 February 2021; Accepted: 16 July 2021; Published online: 09 August 2021

\section{REFERENCES}

1. Abbe, E. Beiträge zur Theorie des Mikroskops und der mikroskopischen Wahrnehmung. Arch. für. Mikroskopische Anat. 9, 413-468 (1873).

2. Rayleigh, L. XXXI. Investigations in optics, with special reference to the spectroscope. Lond. Edinb. Dublin Philos. Mag. J. Sci. 8, 261-274 (1879).

3. Hell, S. W. \& Wichmann, J. Breaking the diffraction resolution limit by stimulated emission: stimulated-emission-depletion fluorescence microscopy. Opt. Lett. 19, 780-782 (1994).

4. Dertinger, T., Colyer, R., lyer, G., Weiss, S. \& Enderlein, J. Fast, background-free, 3D super-resolution optical fluctuation imaging (SOFI). Proc. Natl Acad. Sci. USA 106, 22287-22292 (2009).

5. Schwartz, O. et al. Superresolution microscopy with quantum emitters. Nano Lett. 13, 5832-5836 (2013).

6. Classen, A., von Zanthier, M. O., Scully, J. \& Agarwal, G. S. Superresolution via structured illumination quantum correlation microscopy. Optica 4, 580-587 (2017).

7. Classen, A., von Zanthier, J. \& Agarwal, G. S. Analysis of super-resolution via 3D structured illumination intensity correlation microscopy. Opt. Express 26, 27492-27503 (2018).

8. Shih, Y. An Introduction To Quantum Optics: Photon And Biphoton Physics (CRC press, 2018). 
9. Giovannetti, V., Lloyd, S., Maccone, L. \& Shapiro, J. H. Sub-Rayleigh-diffractionbound quantum imaging. Phys. Rev. A 79, 013827 (2009).

10. Xu, D.-Q. et al. Experimental observation of sub-Rayleigh quantum imaging with a two-photon entangled source. Appl. Phys. Lett. 106, 171104 (2015).

11. Unternährer, M., Bessire, B., Gasparini, L., Perenzoni, M. \& Stefanov, A. Superresolution quantum imaging at the Heisenberg limit. Optica 5, 1150-1154 (2018).

12. Toninelli, E. et al. Resolution-enhanced quantum imaging by centroid estimation of biphotons. Optica 6, 347-353 (2019).

13. Boto, A. N. et al. Quantum interferometric optical lithography: exploiting entanglement to beat the diffraction limit. Phys. Rev. Lett. 85, 2733-2736 (2000).

14. Rozema, L. A. et al. Scalable spatial superresolution using entangled photons. Phys. Rev. Lett. 112, 223602 (2014).

15. Giovannetti, V. \& Lloyd, L. M. S. Quantum-enhanced measurements: beating the standard quantum limit. Science 306, 1330-1336 (2004).

16. Polino, E., Valeri, M., Spagnolo, N. \& Sciarrino, F. Photonic quantum metrology. AVS Quantum Sci. 2, 024703 (2020).

17. Tsang, M. Quantum imaging beyond the diffraction limit by optical centroid measurements. Phys. Rev. Lett. 102, 253601 (2009).

18. Pearce, M. E., Mehringer, T., von Zanthier, J. \& Kok, P. Precision estimation of source dimensions from higher-order intensity correlations. Phys. Rev. A 92 043831 (2015).

19. Vlasenko, S. et al. Optimal correlation order in superresolution optical fluctuation microscopy. Phys. Rev. A 102, 063507 (2020).

20. Horodecki, R., Horodecki, P., Horodecki, M. \& Horodecki, K. Quantum entanglement. Rev. Mod. Phys. 81, 865-942 (2009).

21. Pittman, T. B., Shih, Y. H., Strekalov, D. V. \& Sergienko, A. V. Optical imaging by means of two-photon quantum entanglement. Phys. Rev. A 52, R3429-R3432 (1995).

22. Strekalov, D. V., Sergienko, A. V., Klyshko, D. N. \& Shih, Y. H. Observation of twophoton "ghost" interference and diffraction. Phys. Rev. Lett. 74, 3600 (1995).

23. Agafonov, I. N., Chekhova, M. V., Iskhakov, T. S. \& Wu, L.-A. High-visibility intensity interference and ghost imaging with pseudo-thermal light. J. Mod. Opt. 56, 422-431 (2009).

24. Chan, K. W. C., O'Sullivan, M. N. \& Boyd, R. W. High-order thermal ghost imaging Opt. Lett. 34, 3343-3345 (2009).

25. Chen, X.-H. et al. Arbitrary-order lensless ghost imaging with thermal light. Opt Lett. 35, 1166 (2010).

26. Bai, Y. \& Han, S. Ghost imaging with thermal light by third-order correlation. Phys. Rev. A 76, 043828 (2007)

27. Erkmen, B. I. \& Shapiro, J. H. Unified theory of ghost imaging with Gaussian-state light. Phys. Rev. A 77, 043809 (2008).

28. Moreau, P.-A. et al. Resolution limits of quantum ghost imaging. Opt. Express 26 7528 (2018).

29. Lemos, G. B. et al. Quantum imaging with undetected photons. Nature $\mathbf{5 1 2}$ 409-412 (2014)

30. Lahiri, M., Lapkiewicz, R., Lemos, G. B. \& Zeilinger, A. Theory of quantum imaging with undetected photons. Phys. Rev. A 92, 013832 (2015).

31. Wang, L. J., Zou, X. Y. \& Mandel, L. Induced coherence without induced emission. Phys. Rev. A 44, 4614 (1991).

32. Zou, X. Y., Wang, L. J. \& Mandel, L. Induced coherence and indistinguishability in optical interference. Phys. Rev. Lett. 67, 318 (1991)

33. Skornia, C., von Zanthier, J., Agarwal, G. S., Werner, E. \& Walther, H. Nonclassical interference effects in the radiation from coherently driven uncorrelated atoms. Phys. Rev. A 64, 063801 (2001).

34. Thiel, C. et al. Quantum imaging with incoherent photons. Phys. Rev. Lett. 99 133603 (2007).

35. Bhatti, D., Classen, A., Oppel, S., Schneider, R. \& von Zanthier, J. Generation of N00N-like interferences with two thermal light sources. Eur. Phys. J. D. 72, 191 (2018).

36. Cabrillo, C., Cirac, J. I., Garcia-Fernandez, P. \& Zoller, P. Creation of entangled states of distant atoms by interference. Phys. Rev. A 59, 1025 (1999).

37. Brainis, E. Quantum imaging with $\mathrm{N}$-photon states in position space. Opt. Express 19, 24228-24240 (2011).

38. Opatrny`, T., Kurizki, G. \& Welsch, D.-G. Improvement on teleportation of continuous variables by photon subtraction via conditional measurement. Phys. Rev. A 61, 032302 (2000).

39. Olivares, S., Paris, M. G. A. \& Bonifacio, R. Teleportation improvement by inconclusive photon subtraction. Phys. Rev. A 67, 032314 (2003).

40. Ourjoumtsev, A., Dantan, A., Tualle-Brouri, R. \& Grangier, P. Increasing entanglement between Gaussian states by coherent photon subtraction. Phys. Rev. Lett. 98, 030502 (2007)

41. Paúr, M. et al. Tempering Rayleigh's curse with PSF shaping. Optica 5, 1177-1180 (2018)
42. Lupo, C., Huang, Z. \& Kok, P. Quantum limits to incoherent imaging are achieved by linear interferometry. Phys. Rev. Lett. 124, 080503 (2020).

43. Tsang, M., Nair, R. \& Lu, X.-M. Quantum theory of superresolution for two incoherent optical point sources. Phys. Rev. X 6, 031033 (2016)

44. Tsang, M. Subdiffraction incoherent optical imaging via spatial-mode demultiplexing. N. J. Phys. 19, 023054 (2017).

45. Len, Y. L., Datta, C., Parniak, M. \& Banaszek, K. Resolution limits of spatial mode demultiplexing with noisy detection. Int. J. Quantum Inf. 18, 1941015 (2020).

46. Motka, L. et al. Optical resolution from Fisher information. Eur. Phys. J. 131, 130 (2016).

47. Mikhalychev, A. B. et al. Efficiently reconstructing compound objects by quantum imaging with higher-order correlation functions. Comm. Phys. 2, 134 (2019).

48. Paúr, M. et al. Reading out Fisher information from the zeros of the point spread function. Opt. Lett. 44, 3114-3117 (2019).

49. Datta, C. et al. Sub-Rayleigh resolution of two incoherent sources by array homodyning. Phys. Rev. A 102, 063526 (2020).

50. Hübel, H. et al. Direct generation of photon triplets using cascaded photon-pair sources. Nature 466, 601-603 (2010).

51. Keller, T. E., Rubin, M. H., Shih, Y. \& Wu, L.-A. Theory of the three-photon entangled state. Phys. Rev. A 57, 2076 (1998).

52. Wen, J., Oh, E. \& Du, S. Tripartite entanglement generation via four-wave mixings: narrowband triphoton w state. J. Opt. Soc. Am. B 27, A11-A20 (2010).

53. Corona, M., Garay-Palmett, K. \& U'Ren, A. B. Experimental proposal for the generation of entangled photon triplets by third-order spontaneous parametric downconversion in optical fibers. Opt. Lett. 36, 190-192 (2011).

54. Corona, M., Garay-Palmett, K. \& U'Ren, A. B. Third-order spontaneous parametric down-conversion in thin optical fibers as a photon-triplet source. Phys. Rev. A $\mathbf{8 4}$ 033823 (2011).

55. Borshchevskaya, N. A., Katamadze, K. G., Kulik, S. P. \& Fedorov, M. V. Three-photon generation by means of third-order spontaneous parametric down-conversion in bulk crystals. Laser Phys. Lett. 12, 115404 (2015).

56. Abouraddy, A. F., Saleh, B. E. A., Sergienko, A. V. \& Teich, M. C. Entangled-photon fourier optics. J. Opt. Soc. Am. B 19, 1174-1184 (2002).

57. Saleh, B. E. A., Teich, M. C. \& Sergienko, A. V. Wolf equations for two-photon light. Phys. Rev. Lett. 94, 223601 (2005).

58. Fisher, R. A. Theory of statistical estimation. In Mathematical Proceedings of the Cambridge Philosophical Society, Vol. 22, 700-725 (Cambridge University Press, 1925).

59. Rao, C. R. Information and accuracy attainable in the estimation of statistical parameters. Bull. Calcutta Math. Soc. 37, 81-91 (1945).

60. Cramer, H. Mathematical Methods of Statistics (Princeton University Press, Princeton, 1946)

\section{ACKNOWLEDGEMENTS}

All the authors acknowledge financial support from the King Abdullah University of Science and Technology (grant 4264.01), A.B.M., I.L.K., and D.S.M. also acknowledge support from the EU Flagship on Quantum Technologies, project PhoG (820365).

\section{AUTHOR CONTRIBUTIONS}

The theory was conceived by A.B.M. and D.S.M. Numerical calculations were performed by A.B.M. The project was supervised by D.S.M., D.L.M., and A.B.M. All the authors participated in the manuscript preparation, discussions, and checks of the results.

\section{COMPETING INTERESTS}

The authors declare no competing interests.

\section{ADDITIONAL INFORMATION}

Correspondence and requests for materials should be addressed to A.B.M.

Reprints and permission information is available at http://www.nature.com/ reprints

Publisher's note Springer Nature remains neutral with regard to jurisdictional claims in published maps and institutional affiliations. 
(c) Open Access This article is licensed under a Creative Commons Attribution 4.0 International License, which permits use, sharing, adaptation, distribution and reproduction in any medium or format, as long as you give appropriate credit to the original author(s) and the source, provide a link to the Creative Commons license, and indicate if changes were made. The images or other third party material in this article are included in the article's Creative Commons license, unless indicated otherwise in a credit line to the material. If material is not included in the article's Creative Commons license and your intended use is not permitted by statutory regulation or exceeds the permitted use, you will need to obtain permission directly from the copyright holder. To view a copy of this license, visit http://creativecommons. org/licenses/by/4.0/.

(c) The Author(s) 2021 